\title{
Assessment of tumor heterogeneity: an emerging imaging tool for clinical practice?
}

\author{
Fergus Davnall • Connie S. P. Yip • Gunnar Ljungqvist • \\ Mariyah Selmi • Francesca Ng • Bal Sanghera • \\ Balaji Ganeshan • Kenneth A. Miles • Gary J. Cook • \\ Vicky Goh
}

Received: 23 July 2012 /Revised: 30 August 2012 / Accepted: 24 September 2012 / Published online: 24 October 2012

(C) The Author(s) 2012. This article is published with open access at Springerlink.com

\begin{abstract}
Background Tumor spatial heterogeneity is an important prognostic factor, which may be reflected in medical images

Methods Image texture analysis is an approach of quantifying heterogeneity that may not be appreciated by the naked eye. Different methods can be applied including statistical-, model-, and transform-based methods.
\end{abstract}

F. Davnall $\cdot$ G. Ljungqvist $\cdot$ M. Selmi $\cdot$ V. Goh

Division of Imaging Sciences and Biomedical Engineering,

King's College London,

London, UK

C. S. P. Yip • V. Goh

Department on Oncology, Guy's \& St Thomas'

NHS Foundation Trust,

London, UK

F. Ng $\cdot$ B. Sanghera

Paul Strickland Scanner Centre, Mount Vernon Hospital,

London, Middlesex, UK

B. Ganeshan · K. A. Miles

Clinical Imaging Sciences Centre,

Brighton and Sussex Medical School,

Brighton, Sussex, Falmer, UK

G. J. Cook

Division of Imaging Sciences \& Biomedical Engineering,

King's College London, PET Imaging Centre,

London, UK

V. Goh $(\bowtie)$

Chair of Clinical Cancer Imaging, Lambeth Wing,

St Thomas Hospital,

Lambeth Palace Road,

London SE1 7EH, UK

e-mail: vicky.goh@kcl.ac.uk
Results Early evidence suggests that texture analysis has the potential to augment diagnosis and characterization as well as improve tumor staging and therapy response assessment in oncological practice.

Conclusion This review provides an overview of the application of texture analysis with different imaging modalities, CT, MRI, and PET, to date and describes the technical challenges that have limited its widespread clinical implementation so far. With further efforts to refine its application, image texture analysis has the potential to develop into a valuable clinical tool for oncologic imaging.

Teaching Points

- Tumor spatial heterogeneity is an important prognostic factor.

- Image texture analysis is an approach of quantifying heterogeneity.

- Different methods can be applied, including statistical-, model-, and transform-based methods.

- Texture analysis could improve the diagnosis, tumor staging, and therapy response assessment.

Keywords Texture analysis · Fractal analysis $\cdot$ Cancer $\cdot C T$. MRI · PET

\section{Introduction}

Tumor heterogeneity

Imaging is used widely in oncologic practice for lesion characterization, confirmation of diagnosis, staging, treatment planning, targeting therapy, assessing treatment response, and surveillance. Diagnosis and staging are typically based 
on a lesion's anatomical appearance and the extent of tumor spread on imaging. Different imaging modalities, such as Xray, ultrasound (US), computed tomography (CT), magnetic resonance imaging (MRI), and positron emission tomography (PET), can be used singly or in combination, depending on the tumor type, site, and clinical question to be answered. A limitation that applies to all imaging modalities is that image intepretation is based on a visual process. Yet, there are features within each image that may not be appreciated readily by the naked eye. Furthermore, when images are analyzed in a more quantitative manner, standard region of interest analysis may provide a mean parameter value, e.g., Hounsfield unit (HU) on CT, signal intensity (SI) on MRI, or standardized uptake value (SUV) on PET, but does not typically describe the underlying spatial distribution.

Tumors are heterogeneous both on genetic and histopathological levels (Fig. 1) with intratumoral spatial variation in the cellularity, angiogenesis, extravascular extracellular matrix, and areas of necrosis. Tumors with high intratumoral heterogeneity have been shown to have poorer prognosis, which could be secondary to intrinsic aggressive biology or treatment resistance [1-3]. It is difficult to assess intratumoral heterogeneity with random sampling or biopsy as this does not represent the full extent of phenotypic or genetic variation within a tumor. Thus, a non-invasive method of assessing the heterogeneity within a tumor might be of clinical benefit, particularly in this age of personalized medicine, to select poor prognosis patients for more intensive therapy. Hence, tumor heterogeneity is a clinically relevant parameter for imaging that may be quantifiable and that could augment standard reporting methods.

\section{Texture analysis}

Texture analysis refers to a variety of mathematical methods that can be used to evaluate the gray-level intensity and position of the pixels within an image to derive so-called 'texture features' that provide a measure of intralesional heterogeneity [4]. Different methods have been applied, including statistical-, model-, and transform-based methods [5-12]. Statistical-based techniques have been most commonly applied and describe the distribution and relationships of graylevel values in the image. Three orders of parameters are described in statistical-based texture analysis. First-order statistics relate to gray-level frequency distribution within the region of interest, which can be obtained from the histogram of pixel intensities [13]. It is dependent on a single pixel value rather than its interaction with neighboring pixels. First-order statistics, based on histogram analysis, include mean intensity, maximum intensity, minimum intensity, uniformity (uniformity of gray-level distribution), entropy (irregularity of graylevel distribution), standard deviation of the gray-level histogram distribution, skewness (asymmetry of the histogram), and kurtosis (flatness of the histogram) (Appendix). Secondorder statistics are co-occurrence measurements calculated using spatial gray-level dependence matrices. These matrices determine how often a pixel of intensity $i$ finds itself within a certain relationship to another pixel of intensity $j$. Second-
Fig. 1 Non-small-cell lung cancer showing spatial variation in staining for angiogenesis (CD34), pimonidazole (hypoxia), and glucose transporter protein expression (Glut-1)

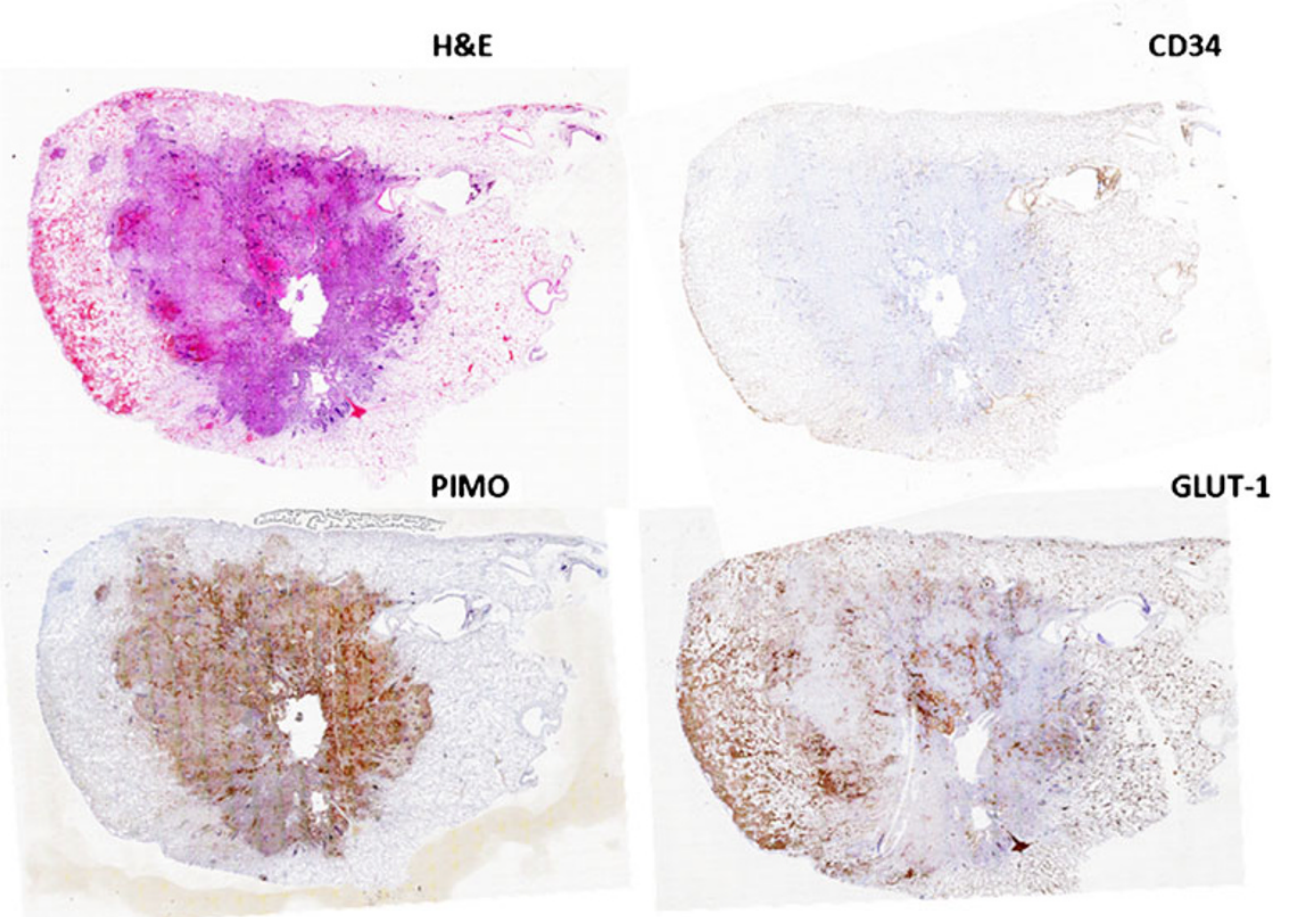


order statistics based on a co-occurrence matrix (GLCM) include entropy (randomness of the matrix), energy/angular second moment (pixel repetition/orderliness and measures the homogeneity of an image), homogeneity (uniformity of cooccurrence matrix), dissimilarity (measurement of how different each element in the matrix is), and correlation (measurement of gray-tone linear dependencies). Another method to derive second-order statistics is the run-length matrix (RLM), which analyzes texture in a specific direction. A run is a length of consecutive pixels with the same gray-level intensity in a preset direction. The relationships between the run lengths give rise to texture. Fine texture has more short runs with similar gray-level intensities, whereas coarse texture has more long runs with different gray-level intensities. Some of the RLM parameters include short-run emphasis (SRE; measures distribution of short runs in an image), long-run emphasis (LRE), gray-level non-uniformity (GLNU; measures similarity of gray-level values; GLNU is small if variation is less), and run length non-uniformity (RLNU; measures the similarity of run lengths; RLN is small if run lengths are similar). Higher-order statistics are calculated using neighborhood gray-tone-difference matrices, which examine the spatial relationship among three or more pixels and are thought to closely resemble the human experience of the image $[14,15]$. This is calculated using the neighborhood gray-tone-difference matrix (NGTDM). Examples of higher-order statistics include contrast (number of local variations within the image), coarseness (measurement of edge density), and busyness (measurement of spatial rate of gray-level change). The application of filters such as Laplacian of Gaussian bandpass filters in statistical-based texture analysis of an image allows the extraction of specific structures corresponding to the width of the filters. Lower filter values (filter 0.5-1.0) will highlight structures with fine textures, and higher filter values highlight structures with medium (filter 1.5-2.0) and coarse (filter 2.5) textures in the filtered image.

Model-based approaches represent texture using sophisticated mathematical models such as fractal analysis. Fractal analysis is a form of pattern or geometric recognition. The fractal dimension is a measurement of the irregularity or roughness of a surface $[13,15]$. Hence, the greater the fractal dimension is, the rougher the texture.

Transform-based methods, such as Fourier, Gabor, and wavelet transforms, analyze texture in a frequency or the scale space. Fourier transform analyzes the frequency content without spatial localization and hence is not often used. Gabor transform is essentially a windowed-Fourier transform derived by the introduction of Gaussian function, which then allows for frequency and spatial localization but is limited by its single filter resolution. This problem is overcome by wavelet transform, which uses multiple channels tuned to different frequencies [15] (Appendix).
Texture analysis is not a new technique and has been studied for medical imaging since 1973, when applied to radiographs, and subsequently to ultrasound [16-18]. More recently, texture analysis has been applied to CT (Figs. 2, 3) and MRI, with an increasing number of PET studies [10, 19-31]. In oncological imaging, texture analysis is reemerging as a potential tool with an increasing number of published studies. A major advantage of texture analysis is that information is maximized from clinical images without the need for additional acquisitions. Studies have focused in several areas: feasibility, technical optimization, validation, and potential clinical applications. This article reviews the the current evidence for texture analysis of CT, MRI, and PET/CT images and the clinical potential in the field of oncology.

\section{Texture analysis of computed tomography images}

Although much of the heterogeneity visible on CT may represent photon noise, which can mask any underlying biological heterogeneity, texture analysis of $\mathrm{CT}$ images has been shown to be feasible by reducing the effect of photon noise while enhancing biological heterogeneity $[6,32]$. A few studies have compared texture features with other imaging and biological parameters (Table 1) [33-35], providing early evidence of potential correlates of CT texture. For example, coarse texture features may reflect the underlying vasculature as defined by CD34 [35]. Nevertheless, further research in this area is still needed.

To date, studies that have been performed have focused in several areas, where the addition of texture to current methods may improve the detection, diagnosis, characterization, and response assessment (Table 2) [5, 32-34; 3643]. By highlighting certain features within a lesion of interest, texture analysis has the ability to improve assessment beyond direct visual analysis by a radiologist.

\section{Diagnosis and characterization}

Several studies have applied various texture analysis methods to improve lesion characterization based on the hypothesis that there are texture differences between benign and malignant lesions. In common, these studies have found that there is greater heterogeneity and higher fractal dimension in tumors than benign lesions, which has the potential to contribute to the computer-aided diagnosis (CAD) of lung or liver lesions. For example, Huang et al. investigated the role of autocovariance function of unenhanced CT images for classifying liver lesions as malignant (80 lesions) or benign ( 84 lesions). The $2 \mathrm{D}$ normalized autocovariance coefficient is a statistical-based texture feature that measures the interpixel correlation within an image. The authors 
Fig. 2 Texture analysis of contrast-enhanced CT images of a colon cancer with the application of different filters highlighting fine, medium, and coarse textures

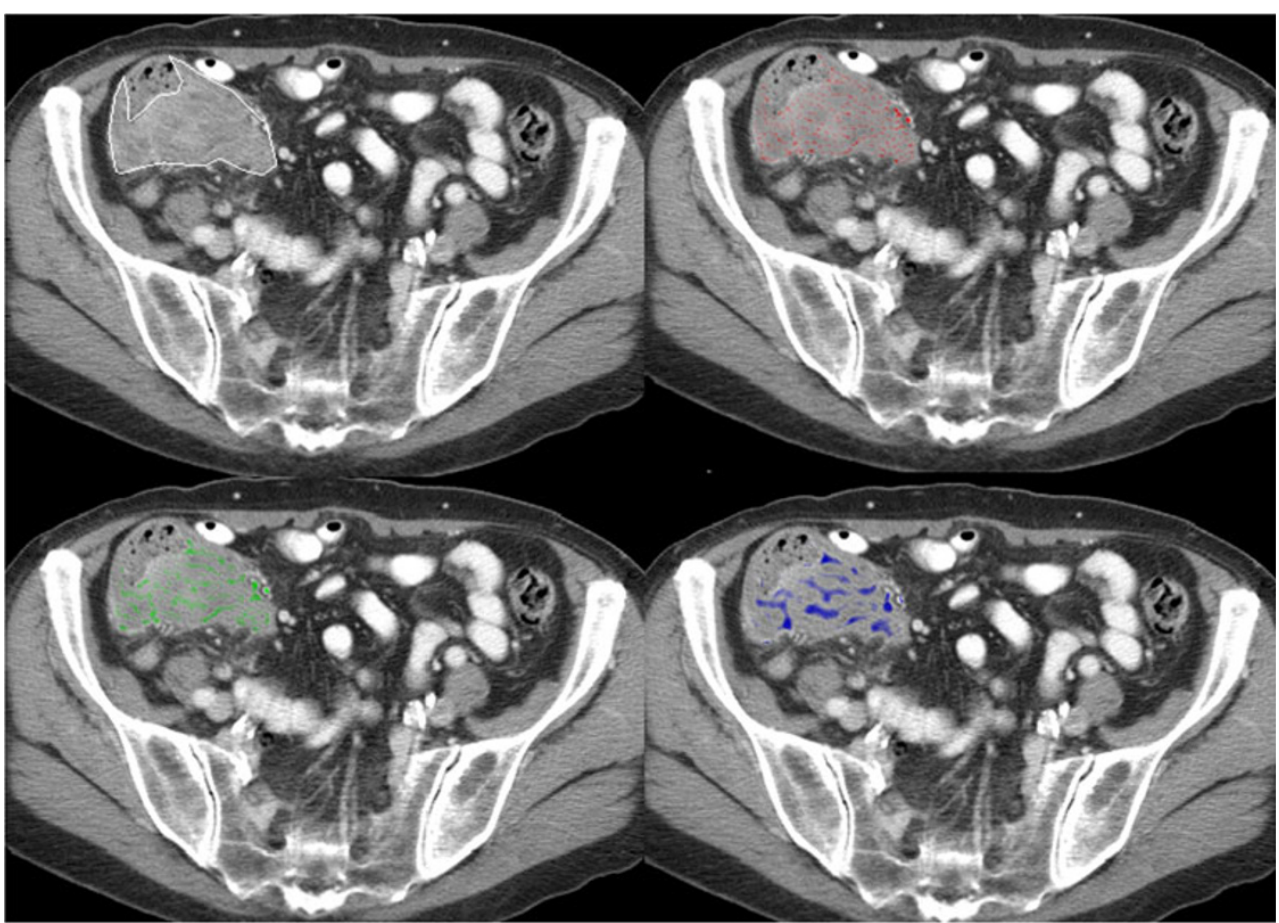

found that its accuracy was $81.7 \%$ for differentiating malignant from benign lesions, but overlap precluded differentiation of primary and secondary tumors [38]. Kido et al. attempted to classify small lung lesions in high-resolution CT images in two separate studies using fractal analysis [36, 37]. In the first study, they evaluated the use of fractal analysis in differentiating benign from malignant lung tumors by comparing either $2 \mathrm{D}$ binary or $3 \mathrm{D}$ gray-level intensity mapping of the fractal dimension in biopsyproven lesions [37]. Benign hamartomas $(n=23)$ had smaller 2D fractal dimensions $(1.17 \pm 0.05)$ compared to bronchogenic carcinomas $(n=70)(1.23 \pm 0.07)$, organizing pneumonias $(n=13)(1.22 \pm 0.07)$, and tuberculomas $(n=11)$ $(1.25 \pm 0.07)(p<0.05)$. However, carcinomas $(2.10 \pm 0.11)$ and hamartomas $(2.12 \pm 0.06)$ had smaller 3D fractal dimensions compared to organizing pneumonias $(2.29 \pm 0.17)$ and
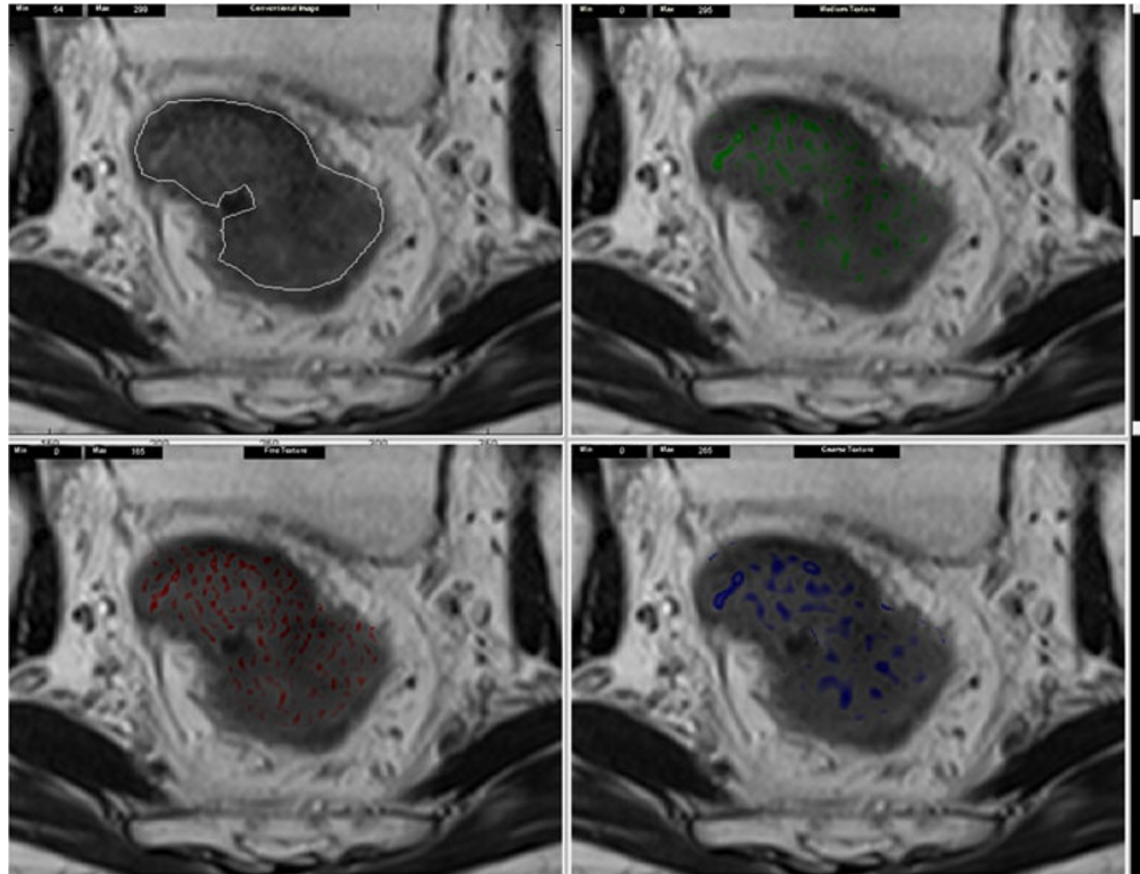
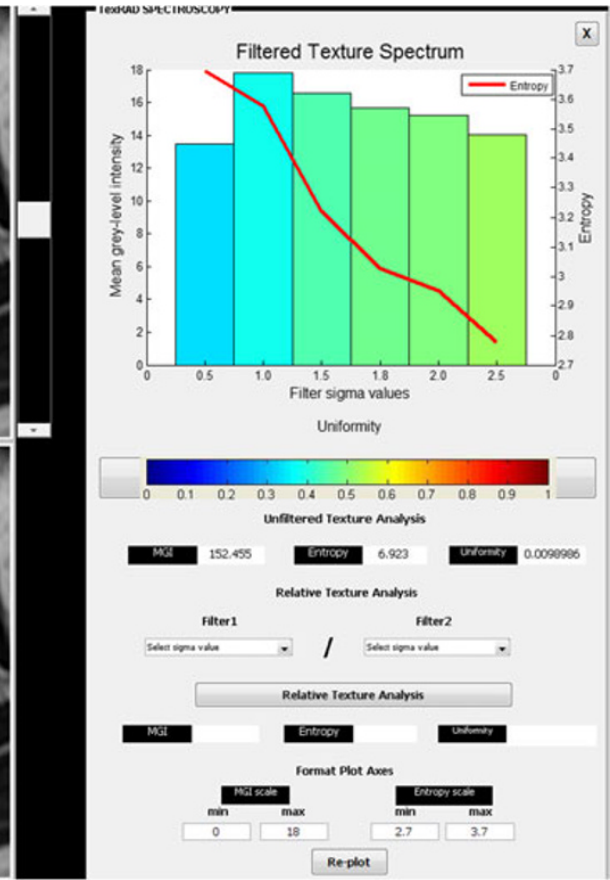

Fig. 3 Texture analysis of a T2-weighted MRI image of rectal cancer 
Table 1 Studies correlating texture features to other imaging and biological parameters
${ }^{\text {a }} S D$ standard deviation of the histogram

\begin{tabular}{|c|c|c|c|}
\hline $\begin{array}{l}\text { Cancer } \\
\text { type }\end{array}$ & Features investigated & Correlate & Author, year \\
\hline Esophagus & $\begin{array}{l}\text { Non-contrast CT. Coarse } \\
\quad \text { texture uniformity } \\
\quad(r=-0.754, \mathrm{p}<0.0001) \\
\text { Entropy }(r=0.748, p=0.0001)\end{array}$ & $\mathrm{SUV}_{\text {mean }}$ & Ganeshan et al., 2012 [33] \\
\hline NSCLC & $\begin{array}{l}\text { Contrast-enhanced CT } \\
\text { Fractal dimension }\end{array}$ & $\mathrm{SUV}_{\text {max }}$ & Al-Kadi et al., 2008 [12] \\
\hline NSCLC & $\begin{array}{l}\text { Non-contrast CT } \\
\text { Coarse texture } \\
\text { Uniformity }(r=-0.52, p=0.03) \\
\text { Entropy }(r=0.51, p=0.03)\end{array}$ & $\mathrm{SUV}_{\max }$ & Ganeshan et al., 2008 [44] \\
\hline NSCLC & $\begin{array}{l}\text { Contrast-enhanced CT } \\
\text { Medium and coarse texture; } \\
\quad \mathrm{SD}^{\mathrm{a}}(r=-0.579, p<0.001) \\
\quad(r=0.591, p<0.001)\end{array}$ & $\begin{array}{l}\text { Histological: CD34 } \\
\text { and pimonidazole }\end{array}$ & Ganeshan et al., 2012 [35] \\
\hline
\end{tabular}

tuberculomas $(2.25 \pm 0.08)(p<0.0001)$. The 3D fractal dimension also differentiated adenocarcinomas $(n=61)$ from squamous cell carcinomas $(n=9)$ [37]. In the second study, the authors found that bronchoalveolar carcinomas $(n=30)$ had greater fractal dimensions compared to nonbronchoalveolar carcinomas $(n=40)$ [36]. Al-Kadi et al. compared fractal analysis of dynamic contrast-enhanced CT (wash-in/wash-out) images. The mean fractal dimension was higher in aggressive/advanced stage tumors compared to non-aggressive/early stage tumors with $83.3 \%$ accuracy for distinguishing between these two groups [5]. Goh et al. found that the fractal dimension and fractal abundance were higher for colorectal tumors compared to normal colon, mean fractal dimension, and abundance (standard deviation): $1.71(0.07)$ and $7.82(0.62)$, respectively, for tumor and $1.61(0.07)$ and $6.89(0.47)$, respectively, for normal colon ( $p \leq 0.001$ ) (Fig. 4) [8]. Cui et al. studied 220 nodes in colorectal cancer and suggested that CT texture features of malignant and benign nodes differed, with greater heterogeneity noted in malignant nodes with a predictive accuracy of $88 \%$ [39]. In a study that included 44 patients with gliomas, Skogen et al. found that coarse texture entropy and uniformity could distinguish between low- and high-grade tumors. Entropy $>5.2$ had a sensitivity and specificity of $76 \%$ and $82 \%$, respectively; uniformity $\leq 0.025$ had a sensitivity and specificity of $64 \%$ and $95 \%$, respectively, for high-grade tumors [40].

\section{Therapy response assessment}

To date, few studies have assessed the potential of texture analysis for response assessment (Figs. 5, 6, 7). Goh et al. investigated the potential of texture analysis to improve response assessment in renal cell cancer metastases treated with tyrosine kinase inhibitors. This study found that texture analysis was a better predictor of response than current response assessment methods based on size and/or enhancement change (RECIST and modified Choi). The percentage changes from baseline values of texture features after two cycles of TKI therapy for metastatic renal cancer were correlated with measured time to progression. Using a threshold identified by ROC analysis, the percentage change of $-2 \%$ or less from baseline for uniformity at a filter value of 2.5 , disease-free survival was significantly better in the group with greater than $-2 \%$ change in uniformity $(p=$ 0.008 ) and performed better than standard response assessment after two cycles of TKI therapy [41].

Relationship with clinical outcome: A potential prognostic biomarker?

Several studies have assessed the potential of texture analysis to improve the prognostic information of current imaging and confirm the hypothesis that greater tumor heterogeneity is an indicator of poor clinical prognosis [33, 42-44]. Texture analysis as a post-processing tool may complement the prognostic information obtained from standard imaging.

Ganeshan et al. found that coarse texture entropy on CT in seemingly normal liver of patients with colorectal cancer correlated with patient survival and postulated that this may be related to micrometastasis formation [42]. The authors also investigated patients with colorectal metastases. They normalized finer texture values (filter 1.0-2.0) to the corresponding texture values obtained from the coarsest filter (filter 2.5) to account for the contribution to the overall texture by the different texture features obtained from different levels of image filtration. It was found that the 
Table 2 Studies investigating the use of CT texture analysis in diagnosis, treatment response assessment, and as a prognostic tool

Diagnosis and characterisation Method Study findings Author, yea

Diagnosis

Lung

Pulmonary nodules

Bronchoalveolar carcinoma vs. non-bronchoalveolar carcinoma

Lung cancer

Liver

Hepatic tumors

GI tract

Colorectal cancer

Colorectal cancer

Brain

Glioma

Response assessment

Metastatic renal cell carcinoma

Prognosis assessment

Liver texture in patients with colorectal cancer but no known metastases

Colorectal cancer metastases

Liver texture in patients with colorectal cancer

Esophageal cancer

NSCLC
Fractal analysis

3D fractal dimension was higher in organizing pneumonias/tuberculomas

Kido et al., 2002 [37]

than carcinomas/hamartomas $(\mathrm{p}<0.001)$

and higher in adenocarcinomas than

squamous cell $(\mathrm{p}<0.05)$

Fractal analysis Fractal dimension higher for bronchoalveolar carcinomas $(2.38 \pm 0.05 / 2.16 \pm 0.01)$ than non- bronchoalveolar carcinomas $(2.19 \pm 0.05 /$

$2.06 \pm 0.01 \mathrm{internal} /$ peripheral; $\mathrm{p}<0.0001$ )

Fractal analysis

Fractal dimension was higher for stage III and

IV cancers than stage I (2.046 vs. 1.534). $83.8 \%$

of stage IV tumors were classified as aggressive

with a threshold of 1.913

Texture analysis Autocovariance function differed between malignant (HCC and colorectal metastases) and benign lesions. Sensitivity of $75.0 \%$ and specificity of $88.1 \%$ were achieved with the proposed diagnostic system

Fractal analysis Fractal dimension and abundance were higher in colon cancer than normal bowel: mean (SD) $1.71(0.07)$ vs. $1.61(0.07)$ for dimension and 7.82(0.62) vs. $6.89(0.47)$ for abundance $(P \leq 0.001)$

Texture analysis Fractal dimension is higher for metastatic nodes

Texture analysis Coarse texture entropy $>5.2$ had a sensitivity and specificity of $76 \%$ and $82 \%$, respectively; uniformity $<0.025$ had a sensitivity and specificity of $64 \%$ and $95 \%$, respectively, for high-grade tumors

Texture analysis

Percentage change in coarse texture uniformity of $\leq-2 \%$ after 2 cycles of TKI correlated with shorter time to progression

Texture analysis Coarse texture entropy correlated with hepatic perfusion index $(r=-0.503978, p=0.007355)$ and survival $(r=0.489642, p=0.009533)$. Hypothesized texture features may reflect vascular changes associated with micrometastases. Entropy $<2.0$ identified patients who died with $100 \%$ sensitivity, $65 \%$ specificity

Texture analysis Uniformity at texture ratios of 1.5/2.5 and 2.0/2.5 were significant OS prognostic factors $(p<0.005)$

Texture analysis Fine texture entropy of $\leq 0.0807$ between $26-30 \mathrm{~s}$ after contrast injection highlighted node-positive patients with $100 \%$ sensitivity, $71 \%$ specificity. HPI did not vary significantly between node-negative and -positive patients

Texture analysis Unenhanced CT component of PET-CT

Greater heterogeneity in higher stage tumors. Coarse uniformity was a significant OS prognostic factor $(O R=4.56,95 \%$ CI $1.08-18.37, p=0.039)$

Texture analysis Coarse texture uniformity $<0.624$ was a poor prognostic factor
Kido et al., 2003 [36]

Al-Kadi et al., 2008 [5]

Huang et al., 2006 [38]

Goh et al., 2007 [8]

Cui et al., 2011 [39]

Skogen et al., 2011 [40]

Goh et al., 2011 [41]

Ganeshan et al., 2007 [42]

Miles et al., 2009 [43]

Ganeshan et al., 2011 [32]

Ganeshan et al., 2012 [33]

Ganeshan et al., 2011 [34] 
Fig. 4 Dynamic contrastenhanced CT (perfusion CT) blood flow parametric map (a); 2D image (b); segmented and thresholded image (c) for fractal analysis

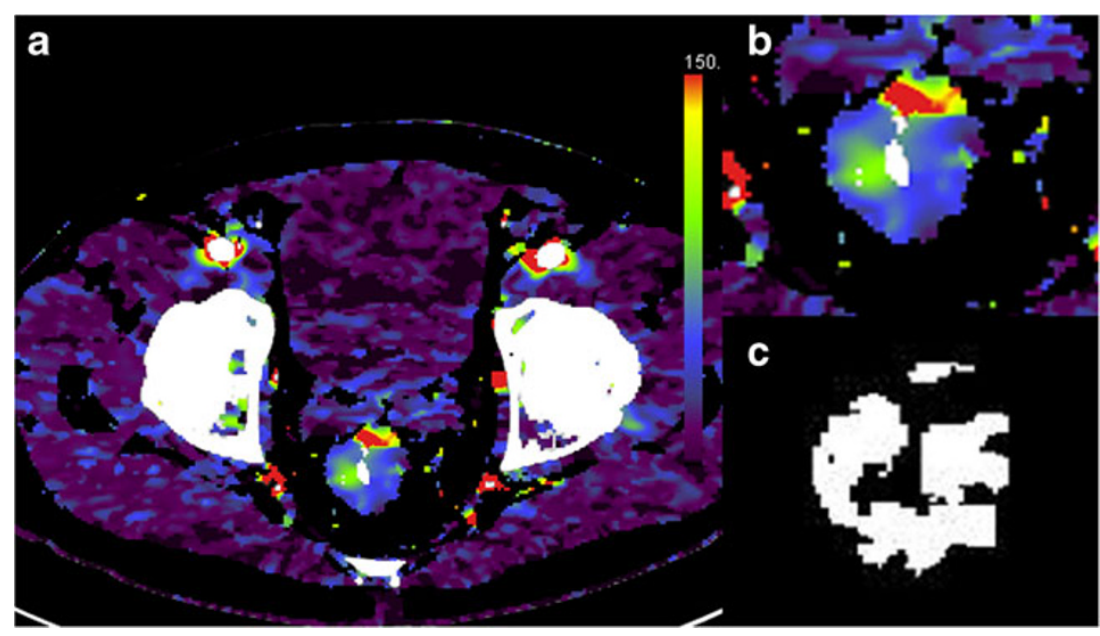

uniformity of surrounding 'normal' liver at texture ratios of 1.5/2.5 and 2.0/2.5 predicted for survival, potentially related to differences in vascularization and shunting related to the presence of metastases [43]. The same group also assessed the texture of dynamic contrast-enhanced CT of the liver in node-negative and -positive non-metastatic colorectal cancer. Uniformity and entropy were significantly different between the node-positive and -negative patients and greatest for fine texture entropy between 26 and $30 \mathrm{~s}$ following injection of intravenous contrast in comparison with the hepatic perfusion index, which was not significantly different between the two groups [32]. In a study of 54 patients with non-small-cell lung cancer (NSCLC) undergoing PETCT staging, a heterogeneous texture on the non-contrastenhanced CT component of the PET-CT was a predictor of poorer survival; in particular, patients with coarse texture uniformity $<0.624$ did not survive more than 2.5 years [44]. Similarly, in a study of 21 patients with primary esophageal cancer undergoing PET-CT staging, advanced stage tumors demonstrated greater heterogeneity at filter values 1.5-2.0. Survival was also poorer for more heterogeneous tumors, particularly for coarse texture uniformity $<0.8477$ (odds ratio $=4.45,95 \%$ CI $1.08-18.37, p=0.039$ ) [33].

\section{Texture analysis of magnetic resonance imaging}

Texture analysis has also shown promise in MRI. The majority of literature over the past 10 years has been directed toward lesion detection and lesion classification, for example, breast, brain, liver, and prostate (Table 3) [19-23, 26, $45-48]$.

\section{Diagnosis and characterization}

As with CT, MRI studies have found that texture features may differ between benign and malignant lesions and may have potential in $\mathrm{CAD}$. In the breast $2 \mathrm{D}$ co-occurrence matrix features of dynamic contrast-enhanced MRI images and signal enhancement ratio maps, $3 \mathrm{D}$ and $4 \mathrm{D}$ features may distinguish between benign and malignant breast lesions: $4 \mathrm{D}$ techniques may achieve a performance similar to human observers (AUC 0.99) [26, 46-48]. Holli et al. have investigated differences in texture between invasive lobular carcinoma (ILC) and invasive ductal carcinoma (IDC), two common but distinct types of breast cancer, using different texture methods. In this study, co-occurrence matrix features were significantly different between ILC and IDC, allowing differentiation between these two histological subtypes, and were superior to the other texture methods applied including histogram analysis, run-length matrix, autoregressive model, and wavelet transform [22].

In the brain, studies have found that texture features of MRI including dynamic contrast-enhanced (DCE) sequences may distinguish between types of tumors [19, 23]. In the first study by Eliat et al., the addition of MRI texture analysis to dynamic contrast-enhanced MRI (DCE-MRI) was able to discriminate glioblastoma multiforme (GBM) from malignant glioneuronal tumors (MGNT) [19]. This study analyzed the use of both first-order and second-order statistics, which included GLCM and RLM methods. This study found that the addition of second-order statistics such as run-length non-uniformity, gray-level non-uniformity, angular second moment, and entropy to the findings from DCE-MRI had $100 \%$ negative predictive value, $79 \%$ positive predictive value, $100 \%$ sensitivity, and $62 \%$ specificity in differentiating MGNT from GBM. Another group developed a computer-assisted classification method combining conventional MRI and perfusion MRI texture analysis using Gabor transform and its implementation as a diagnostic tool [23]. When the method was applied to 102 different brain tumors, including metastasis $(n=24)$, meningiomas $(n=4)$, grade II gliomas $(n=22)$, grade III gliomas $(n=18)$, and glioblastomas $(n=34)$, the accuracy, sensitivity, and specificity achieved by this method were $85 \%, 87 \%$, and $79 \%$, respectively, for discrimination of metastases 
Fig. 5 Changes in texture features of esophageal cancer following neoadjuvant chemotherapy: baseline (a) and following chemotherapy (b). An increase in homogeneity is noted with treatment
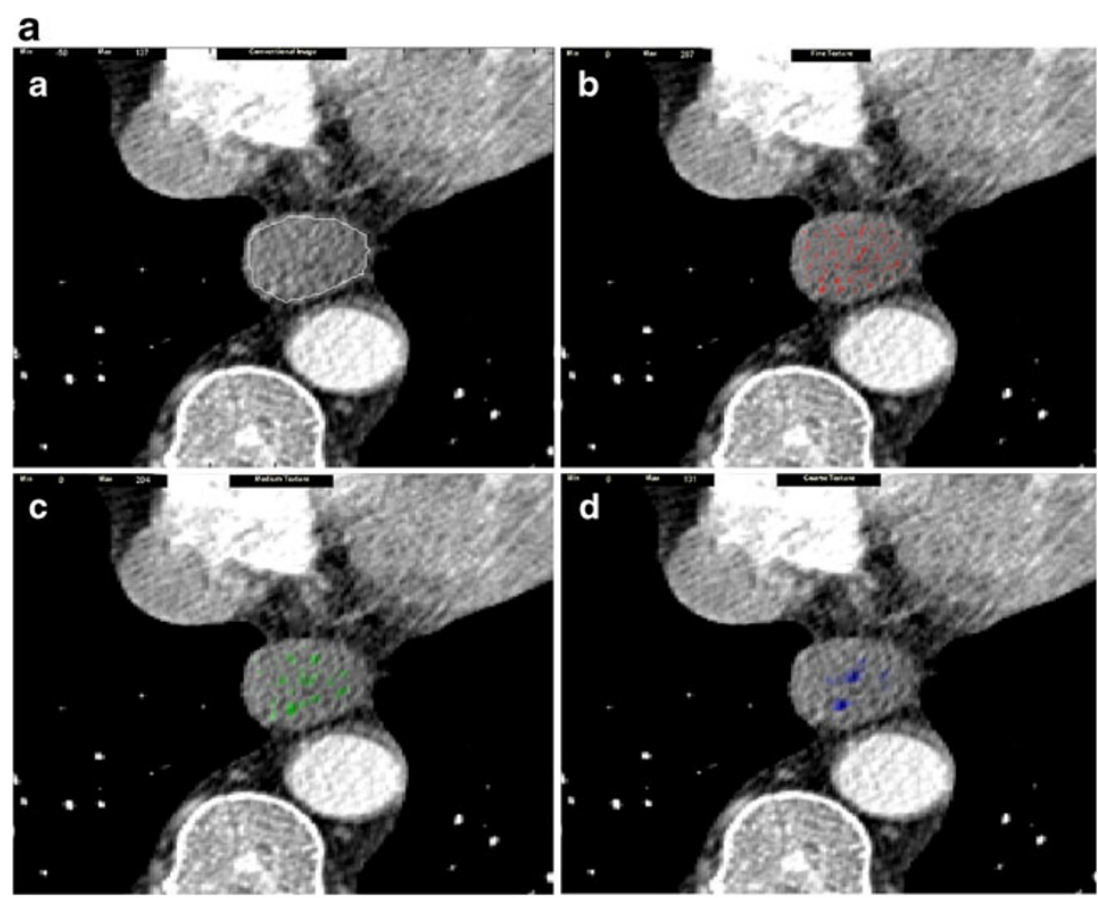

b
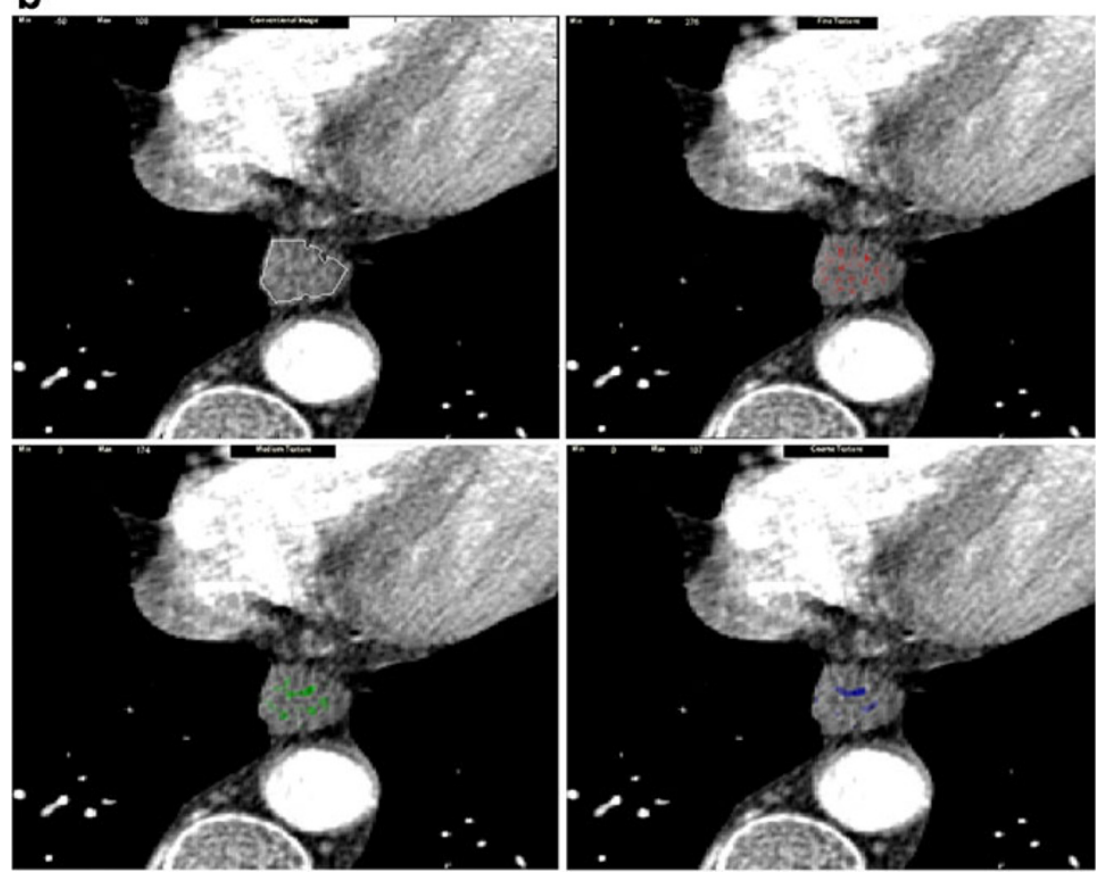

from gliomas and $88 \%, 85 \%$, and $96 \%$ for discrimination of high-grade (grades III and IV) from low-grade (grade II) neoplasms.

In the liver, an exploratory study on unenhanced T1- and T2-weighted MRI showed that it was feasible to use texture analysis to classify benign cysts and hemangiomas, though with up to $25 \%$ misclassified [20]. This study used firstand second-order statistics (GLCM and RLM) and also the wavelet transform method to derive texture parameters that were then selected based on their discriminative value in differentiating cysts from haemangiomas and subsequently used in the computer-assisted classification algorithm. Two prostate studies have shown the potential of fractal features in distinguishing between benign and malignant disease with histological confirmation [21, 49]. For example, Lv et al. investigated the use of the texture fractal dimension (TFD) and histogram fractal dimension (HFD) based on the box-counting method and histogram fractal analysis of the intensity distribution, respectively. The mean and standard deviations of TFD and HFD for cancerous and non- 


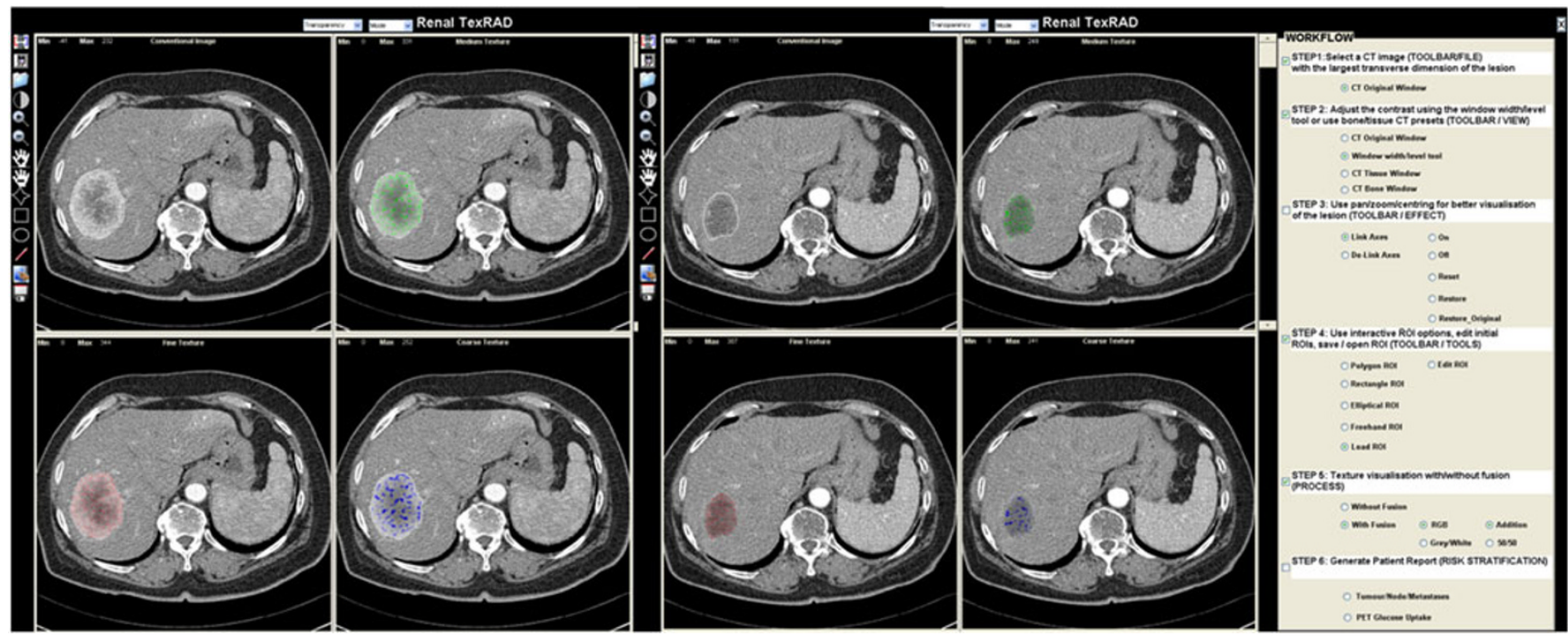

Fig. 6 Changes in texture features of metastatic renal cancer following two cycles of a tyrosine kinase inhibitor: baseline (left) and following therapy (right). An increase in homogeneity is noted with treatment

cancerous lesions were significantly different (TFD: $2.76 \pm$ 0.11 vs. $2.81 \pm 0.15, p=0.035$; HFD: $1.23 \pm 0.05$ vs. $1.42 \pm$ $0.09, p<0.001)$. The authors also showed that an area under the ROC curve of 0.96 could be achieved for the histogram fractal dimension in a cohort of 55 patients who had diagnoses confirmed by ultrasound-guided biopsy [49].

\section{Therapy response assessment}

Studies of response assessment have shown that assessment of heterogeneity is feasible (Fig. 5), may augment response assessment, and is a predictor of response [24, 27, 28].
Harrison et al. demonstrated that MRI texture appearances change during treatment in 19 patients with non-Hodgkin lymphoma who were imaged with $\mathrm{T} 1$ and $\mathrm{T} 2 \mathrm{~W}$ MRI at three time points: at staging, and after the first and fourth cycle of chemotherapy [27]. Alic et al. examined the role of MRI texture in response prediction following isolated limb perfusion in unresectable soft tissue sarcoma of the extremities. They showed that responding tumors demonstrated high coherence in the pre-treatment MRI, a texture parameter that measures how spatially close the high intensity voxels are to each other [24]. Similarly, O'Connor et al. demonstrated in 10 patients with 26 liver metastases from
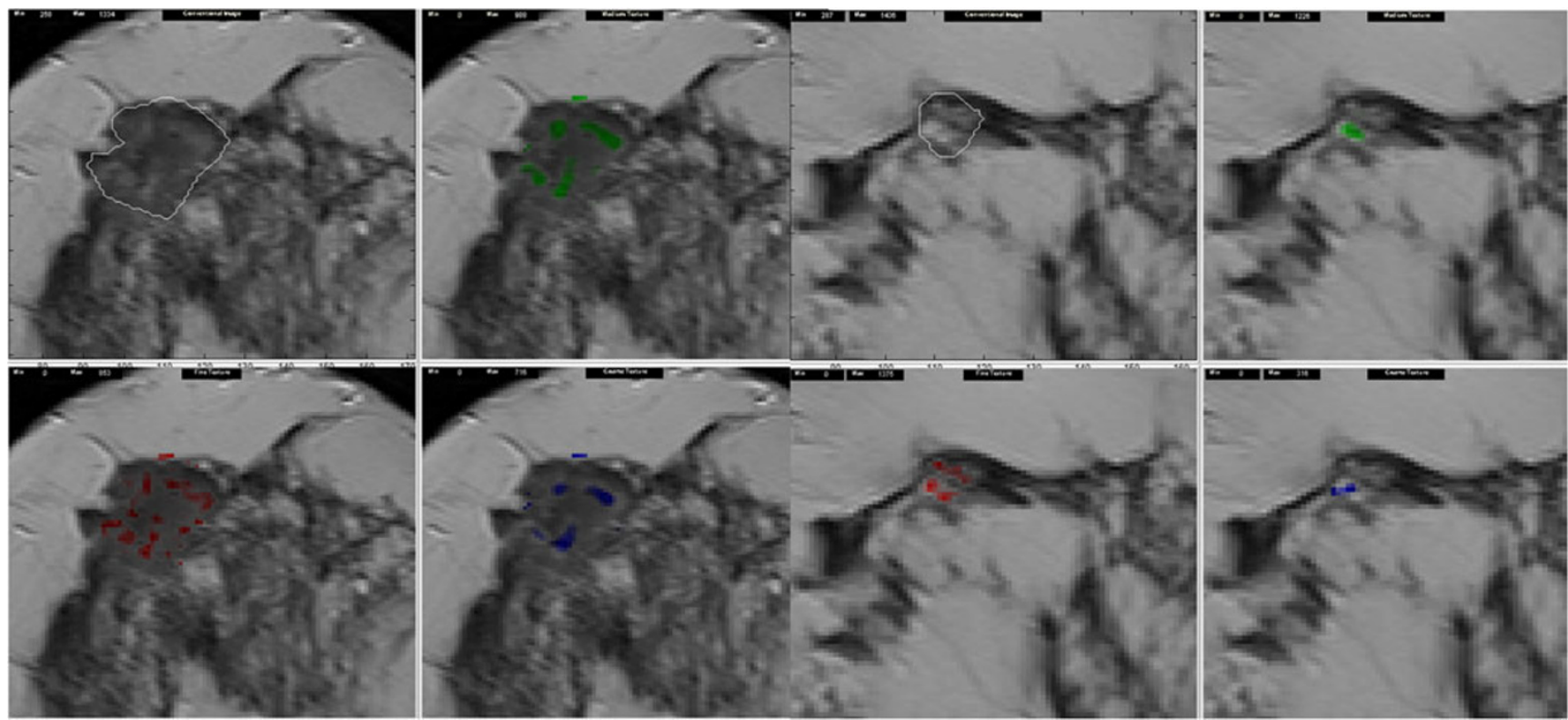

Fig. 7 Changes in breast tumor texture following neoadjuvant chemotherapy on T2W-weighted MRI. Tumor shrinkage and an increase in homogeneity are noted following completion of chemotherapy (right) 
Table 3 Studies investigating the use of MRI texture analysis in diagnosis, treatment response assessment, and as a prognostic tool

\begin{tabular}{|c|c|c|}
\hline Diagnosis and characterization & Method & Study findings \\
\hline
\end{tabular}

Diagnosis

\section{Breast}

Simulated microcalcification

Breast cancer

Texture analysis

Breast lesion

Breast cancer

Invasive lobular and

ductal breast cancer

Brain

Glioneuronal tumor

Brain tumors - metastases, meningiomas, gliomas (grade II and III), glioblastomas

Prostate

Prostate cancer

Prostate cancer

Fractal analysis

Liver

Liver cysts and hemangiomas

Texture analysis

\section{Response assessment}

Breast

Texture analysis

Texture analysis

Texture analysis

Texture analysis

Texture analysis

Texture analysis

Fractal analysis

Texture analysis
The combination of fractal and multifractal features was more accurate than classical texture features in detecting cancer and was more robust against signal intensity variations

Both fractal analyses offered promising quantitative indices for prostate cancer identification, with histogram fractal dimension offering a more robust diagnosis than texture fractal analysis (correlation coefficient of $c=0.9905$ vs. $c=0.9458$, respectively)

Texture analysis (first-order, second-order statistics and wavelet transform) was suc cessfully used to classify focal liver lesions on zero-fill interpolated 3.0-T MR images
Second-order statistics extracted from parametric maps that reflect lesion washout properties discriminate malignant from benign tumors better than textural features extracted from either first postcontrast frame lesion area or from parametric
James et al., 2001 [45]

Gibbs et al., 2003 [46]

Chen et al., 2007 [47]

Woods et al.,2007 [48]

Holli et al., 2010 [22]

Eliat et al., 2012 [19]

Zacharaki et al., 2009 [23]

Lopes et al., 2011 [21]

Lv et al., 2009 [49]

Mayerhoefer et al., 2010 [20]

Karahaliou et al., 2010 [26] 
Table 3 (continued)

\begin{tabular}{|c|c|c|c|}
\hline Diagnosis and characterization & Method & Study findings & Author, year \\
\hline & & $\begin{array}{l}\text { map reflecting lesion initial uptake. Angular } \\
\text { second moment and entropy were most dis } \\
\text { criminative }\end{array}$ & \\
\hline Lymphoma & Texture analysis & $\begin{array}{l}\text { Texture analysis [first-order, second-order } \\
\text { statistics (GLCM and RLM), autoregressive } \\
\text { model and wavelet transform] was able to } \\
\text { classify NHL lesions undergoing chemo } \\
\text { therapy based on changes following treatment }\end{array}$ & Harrison et al.,2009 [27] \\
\hline Liver metastases & Fractal analysis & $\begin{array}{l}\text { Tumor heterogeneity as assessed by fractal } \\
\text { dimension predicted tumor shrinkage in } \\
\text { response to bevacizumab and cytotoxic } \\
\text { chemotherapy in colorectal liver metastases }\end{array}$ & O’Connor et al., 2011 [28] \\
\hline
\end{tabular}

colorectal cancer treated with bevacizumab and FOLFOX-6 chemotherapy that fractal measures derived from pre-treatment DCE-MRI were associated with tumor response [28].

\section{Texture analysis of positron emission tomography (PET)}

At present, only a few studies have investigated the potential of PET texture analysis. These studies have focused on its prediction of outcome and potential for radiotherapy planning.

\section{Prediction of outcome}

To date, three studies have been published. Eary et al. showed that image heterogeneity as assessed by a new method using a heterogeneity variable (HET), which was defined as the percentage of variability in the voxel-level FDG uptake compared to an 'ideal' ellipsoidal upake pattern, was a valid prediction method in patients with sarcoma. An increase of $6.5 \%$ in heterogeneity was associated with a $65 \%$ increase risk of death, the risk being higher in patients with high-grade disease [50]. El Naqa et al. demonstrated that various first- and second-order statistical textural features are useful in predicting outcome in head and neck ( $n=$ 9) and cervical cancer $(n=14)$. The authors combined the various first- and second-order statistics (energy, contrast, local homogeneity, and entropy) with features indicating the shape of the tumors in a linear regression model to predict treatment response to chemoradiation. These methods achieved an AUC of 0.76 and 1.0 for the cervix and head and neck cohorts, respectively [30]. Tixier et al. have investigated its clinical application in 41 patients with esophageal cancer treated with chemoradiation and shown that baseline FDG PET texture is a sensitive predictive marker. They found that local (i.e., entropy and homogeneity) and regional (i.e., size and intensity variabilities) texture parameters performed better than standard SUV measurements in identification of responders from non-responders following chemoradiation. The sensitivity, specificity, and AUC for SUVmax were $53 \%, 73 \%$, and 0.59 compared to $73 \%$, $88 \%$, and 0.89 for local homogeneity in identifying responders [31].

Potential for radiotherapy planning

$\mathrm{Yu}$ et al. have assessed whether first-order, second-order, and higher-order statistics in FDG PET-CT co-registered images can differentiate between normal and abnormal nodes to assist radiotherapy target planning. Abnormal nodes were found to be more heterogeneous than normal tissues in PET images and, of interest, have higher uniformity in CT images [51]. It could be that the accuracy of texture analysis based on non-contrast enhanced CT scan is lower than PET texture or that there was indeed a true difference between CT and PET textures when abnormal tissues are compared to the normal tissues. However, the authors found that a combination of PET and CT textures, particularly second-order and higher-order statistics, had higher discriminative power. This group subsequently developed an automated radiotherapy volume delineation software ("COMPASS") based on their findings from the initial study. They studied its use in ten patients with head and neck cancer by comparing this to three PET segmentation methods: threshold SUV value of 2.5, threshold of $50 \%$ maximal intensity and signal/background ratio, as well as tumor volume delineation by three independent radiation oncologists. This study found that automated texture-based segmentation correlated better with tumor delineation by oncologists compared to PET segmentation [52].

\section{Technical challenges for clinical implementation of texture analysis}

Although studies so far have shown clinical promise, there are still technical considerations to contemplate, particularly 
the effect of image acquisition, image quality, texture methods and software platforms on parameter values, the need for harmonization of acquisitions, and a standardized analysis approach for clinical application [5, 12, 32, 53-56]. Each of the modalities brings different challenges to texture analysis. With PET, images are of a lower spatial resolution than CT or MRI. Although there is no specific limit to the size of the lesion that is amenable to this analysis, small tumors such as nodes, which are below the spatial resolution of PET scans, may not be suitable for this technique. With MRI, scanner and sequence acquisition parameters have a greater non-linear influence on signal intensity and quantification of heterogeneity in comparison with CT or PET, thus requiring stringent quality control and physics input.

To date, many studies have focused on a limited tumor area, such as the largest cross-sectional area, rather than the whole tumor volume. Intratumoral heterogeneity is likely to be greater in the whole tumor as compared to a limited region; hence, this could dilute the diagnostic and prognostic value of texture analysis. With region-of-interest delineation around a tumor, this has the potential to introduce inter- and intraobserver variability. If a standardized automated ROI propagation is used, non-tumor areas may be included in the analysis of the pixel values, which may confound the results obtained.

However, the potential impact of this methodical difference on clinical findings is largely unexplored. At present, the reproducibility of texture analysis has yet to be established widely, although a few studies have begun to address issues related to image acquisition and image quality, and their effect on texture analysis [32, 53-56]. For example, Sanghera et al. have demonstrated that the reproducibility of fractal analysis in two scans performed $24 \mathrm{~h}$ apart by a single reader is good, with a mean difference ( $95 \%$ limits of agreement) of $-0.024(-0.212$ to 0.372 ) and -0.355 ( -0.869 to 1.579$)$ for $2 \mathrm{D}$ fractal dimension and fractal abundance, and -0.024 ( -0.307 to 0.355 ) and -0.043 $(-1.154$ to 1.239$)$ for 3D fractal dimension and fractal abundance, respectively [9]. Good interobserver variability is also observed with a mean difference of 0.030 (95\% limits of agreement -0.143 to 0.204$)$ and -0.073 ( -0.823 to 0.676 ) for $2 \mathrm{D}$ and $3 \mathrm{D}$ fractal dimensions, and -0.073 ( -0.823 to 0.676 ) and -0.044 ( -0.139 to 0.052$)$ for $2 \mathrm{D}$ and $3 \mathrm{D}$ fractal abundances, respectively. For therapeutic assessment where repeated imaging is performed, it may be appropriate to use the same scanner and acquisition parameters to ensure consistency. Similarly, if contrast-enhanced images are used, contrast agent administration should be consistent to minimize variability in gray level intensity related to differences in contrast agent administration or dose. Use of a 'texture phantom' would also allow calibration of imaging systems within a multicenter setting. Further work in this area is still needed.

The implementation of texture analysis into the routine clinical workflow will remain a challenge. Although patients are not required to undergo any additional imaging, as texture analysis is a post-processing step that can be performed on existing DICOM format images, at present, such analysis and software remain as research tools, with few commercially available options.

\section{Conclusions}

Although texture analysis is not a new technique, there has been resurgent interest in the assessment of tumor heterogeneity, particularly for CT, MRI, and PET, in the last 10 years, albeit in relatively small studies. Nonetheless, it is showing promise in the diagnosis and characterization of tumors, response assessment, and as a predictive biomarker, which should be explored further in larger prospective studies. Texture analysis maximizes the information obtained from current standard images and can be implemented into the clinical workflow. With further efforts to refine its applications and direct standardization, this technique has the potential to develop into a valuable clinical tool in oncologic imaging in the future.

Acknowledgements B. Ganeshan has a commercial interest in the tumor textural analysis software ('TexRAD'). The authors acknowledge financial support from the Department of Health via the National Institute of Health Research Biomedical Research Centre award to Guy's and St Thomas' NHS Foundation Trust in partnership with King's College London and King's College Hospital NHS Foundation Trust.

Conflicts of Interest Dr. Balaji Ganeshan has a commercial interest in the tumor textural analysis software ('TexRAD').

Funding The authors acknowledge financial support from the Department of Health via the National Institute of Health Research Biomedical Research Centre award to Guy's and St Thomas' NHS Foundation Trust in partnership with King's College London and King's College Hospital NHS Foundation Trust.

Open Access This article is distributed under the terms of the Creative Commons Attribution License which permits any use, distribution, and reproduction in any medium, provided the original author(s) and the source are credited.

\section{Appendix}

Statistical-based approaches applied to CT images

An approach that has been used in recent publications is the application of a Laplacian of Gaussian (LoG) bandpass filter to highlight and enhance different spatial scales between fine and coarse texture (filter value $=1.0$ to 2.5) [1]

The two-dimensional (2D) Gaussian distribution $G$ is given by:

$G(x, y)=e^{-\frac{x^{2}+y^{2}}{2 \sigma^{2}}}$ 
where $x, y$ are the spatial coordinates of the image matrix and $\sigma$ is the standard deviation of the Gaussian distribution.

The 2D Gaussian distribution effectively blurs the image, wiping out all structures at scales much smaller than the sigma of the Gaussian. This distribution has the desirable characteristics of being smooth and localized in both the spatial and frequency domains, and is therefore less likely to introduce any changes to the original image. The Gaussian distribution highlights only texture features of a particular scale. A fine scale $(<4$ pixels) enhances parenchyma, while a medium-to-coarse scale (6-12 pixels) enhances the underlying vasculature.

The reason for using the Laplacian $\left(\nabla^{2}\right)$ is that it is the lowest-order orientation-independent (isotropic) differential operator and inherently has less computational burden and can be used to detect intensity changes in an image that correspond to the zero crossings of the filter. $\nabla^{2} G$ is the Laplacian of Gaussian filter, a circularly symmetric, Mexican-hat-shaped filter whose distribution in the 2D spatial domain is given by

$\nabla^{2} G(x, y)=\frac{-1}{\pi \sigma^{4}}\left(1-\frac{x^{2}+y^{2}}{2 \sigma^{2}}\right) e^{-\left(\frac{x^{2}+y^{2}}{2 \sigma^{2}}\right)}$.

From the mathematical expression of this circularly symmetric filter at different filter values, the number of pixels representing the width between the diametrically opposite zero-crossing points in this filter can be calculated. The width of the filter at different filter values is obtained by evaluating the Laplacian of the Gaussian spatial distribution along the $x$ and $y$ directions. The lower the filter value, the smaller is the filter width in the spatial domain and the larger is the pass-band region of the filter in the frequency domain, highlighting fine details or features in the filtered image in the spatial domain. Similarly in the spatial domain, a higher filter value allows coarse features to be highlighted in the filtered image.

Filtration can be done in the spatial or frequency domain. In the spatial domain, the filter mask is convolved with the image, which involves intensive computation. It is more efficient to use the filter in the frequency domain, as convolution of the filter mask and the image in the spatial domain is equivalent to multiplication of the Fourier transforms of the filter mask and image in the frequency domain. The inverse Fourier transform of the filtered spectrum gives the resultant filtered image in the spatial domain. Also, the accuracy of this filtering operation is improved when used in the frequency domain, as the quantization errors arising from the convolution of the filter, especially for small $\sigma$ values in the spatial domain, would distort the image.

Quantification of CT texture following filtration is typically performed for a specified region of interest (e.g., the largest tumor cross-sectional area) or for the whole tumor.
Thresholds can be applied to the original CT image. In the case of rectal or lung tumors, this may be to exclude surrounding air by removing any pixels with attenuation values below $-50 \mathrm{HU}$ from the analysis. The same ROI or VOI is applied at all filter scales.

Typical parameters derived from the histogram analysis include the kurtosis, skewness, and standard deviation of the pixel distribution histogram, mean gray level intensity, entropy, and uniformity. Kurtosis (or the magnitude of pixel distribution), skewness (or the skewness of the pixel distribution), and the standard deviation of the pixel distribution describe the shape of the histogram representing the peak, asymmetry, and gray-level variation within the lesion. Entropy is a measure of texture irregularity, while uniformity reflects the distribution of gray levels within the tumor.

Higher entropy, lower uniformity, higher standard deviation, higher kurtosis, and positive skewness are thought to represent increased heterogeneity and portend poorer prognosis [2-4].

$s d=\left(\frac{1}{(\mathrm{n}-1)} \sum_{(x, y) \in R}[a(x, y)-\bar{a}]^{2}\right)^{\frac{1}{2}}$

$$
\begin{gathered}
k=\frac{\mathrm{n}(\mathrm{n}+1)}{(\mathrm{n}-1)(\mathrm{n}-2)(\mathrm{n}-3)} \frac{\sum_{(x, y) \in R}[a(x, y)-\bar{a}]^{4}}{[s d(a)]^{4}} \\
-3 \frac{(n-1)^{2}}{(n-2)(n-3)} \\
s=\frac{\mathrm{n}}{(\mathrm{n}-1)(\mathrm{n}-2)} \frac{\sum_{(x, y) \in R}[a(x, y)-\bar{a}]^{3}}{[s d(a)]^{3}}
\end{gathered}
$$

$e=-\sum_{l=1}^{k}[p(l)] \log _{2}[p(l)]$

$u=\sum_{l=1}^{k}[p(l)]^{2}$

Where:

$\bar{a}=\frac{1}{n} \sum_{(x, y) \in R}[a(x, y)]$

$\bar{a}$ is the mean value within $R, R$ is the ROI within the image $a(x, y), n$ is the total number of pixels in $R, I$ is the 
pixel value (between $I=1$ to $k$ ) in $R$, and $p(I)$ is the probability of the occurrence of that pixel value.

\section{Autocorrelation model}

The autocorrelation coefficient denotes the interpixel correlation within an image. This coefficient is modified to a mean-removed version to generate similar autocorrelation features with different brightness but similar texture called the autocovariance coefficient [5]. The autocovariance coefficient between pixel $(i, j)$ and pixel $(i+\Delta m, j+\Delta n)$ in an image of size $M \times N$ is given by:

$\gamma(\Delta m, \Delta n)=\frac{A^{\prime}(\Delta m, \Delta n)}{A^{\prime}(0,0)}$

$$
\begin{gathered}
A^{\prime}(\Delta m, \Delta n)=\frac{1}{(M-\Delta m)(N-\Delta n)} \\
\sum_{x=0}^{M-1-\Delta m} \sum_{y=0}^{N-1-\Delta n}|(f(x, y)-\bar{f})(f(x+\Delta m, y+\Delta n)-\bar{f})|
\end{gathered}
$$

where ${ }^{-} f$ is the average value of $f(x, y)$.

Model-based approaches applied to CT images

\section{Fractal analysis}

Fractal analysis provides a means of assessing structural geometry. Fractal measures such as the fractal dimension (a measure of how an object fills space), fractal abundance (a measure of the volume of space filled), and lacunarity (a measure of the structural heterogeneity within an object) inform about different aspects of the spatial pattern of the tumor vasculature, providing a measure of its spatial heterogeneity. Fractal dimension and fractal abundance may be calculated using a 2D square and 3D cube, the box counting method, with multiple grid offsets for all possible box start locations, based on the following equation:

$\mathrm{N}_{\mathrm{L}}=\mathrm{KL}^{-\mathrm{D}}$

where $\mathrm{L}$ is the box size, $\mathrm{N}_{\mathrm{L}}$ is the number of boxes of size $\mathrm{L}$ needed to cover the object being studied, and $\mathrm{D}$ is the fractal dimension. By plotting a log-log plot of $\mathrm{N}_{\mathrm{L}}$ versus $\mathrm{L}$, fractal dimension (FD) can be obtained from the slope, and fractal abundance (FA) or log K can be obtained from the $\mathrm{y}$ intercept of the straight portion of the curve [6]. The 2D and $3 \mathrm{D}$ techniques have been validated using simple and complex structures of known fractal dimension, e.g., Sierpinski gasket and carpet with multiple iterations, and shown to be reproducible [7].
Lacunarity can be derived using a 2D and modified 3D gliding box method defined by the equation:

$\Lambda=\sum \mathrm{s}^{2} \mathrm{Q}(\mathrm{s}, \mathrm{r}) /\left[\sum \mathrm{sQ}(\mathrm{s}, \mathrm{r})\right]^{2}$

where $\Lambda$ represents lacunarity, $r$ represent box size, s represents the number of occupied sites within a box size, and Q is the probability distribution (representing the frequency distribution of the total number of occupied sites for a box size $r$ over the total number of boxes of size r) [8].

\section{Transform-based approaches applied to CT images}

The pixel pattern in a 2D image has a unique frequency pattern in a specific spatial scale of the image $[9,10]$. If the gray-level values change quickly, it is deemed to have a high spatial frequency. If the gray level values vary slowly such that there is very little variation within an image, it is considered to have a low spatial frequency. This is dependent on the scale used to analyze the image. For example, if a large scale is used, then there is less variation observed compared to the greater variation and details seen with a smaller scale.

\section{Fourier transform}

Fourier transform is useful in the analysis of global frequency content but is without time/space localization [11]. The window Fourier transform of a 1D signal $f(x)$ is given by:

$$
F_{w}(u, \xi)=\int_{\infty}^{\infty} f(x) w(x-\xi) e^{-j 2 \mathrm{p} u x} d x
$$

\section{Gabor transform}

Gabor transform introduces the time dependency element into Fourier analysis by multiplying it with a Gaussian function [i.e., window function $w(x)$ becomes Gaussian] [12]. The time-frequency resolution of the Gabor transform is fixed throughout the time-frequency plane [12]. Gabor output is also non-orthogonal; thus, the resultant texture features may have significant correlation [13].

\section{Wavelet transform}

Multiresolution analysis such as wavelet transform uses multiple channels tuned to different frequencies (i.e., the window function varies). Wavelet transform was first introduced by Mallat [14]. It decomposes an image by using spatially oriented frequency filters but requires intensive computation. Wavelets are considered as a family of 
functions derived from translations and dilations of a single function known as the "mother wavelet" $\Psi(\mathrm{t})$. Wavelets are defined by:

$\psi_{a, b}(t)=\frac{1}{\sqrt{|a|}} \psi\left(\frac{t-b}{a}\right), \quad a, b \in R, \quad a \neq 0$

where $a$ is the scaling parameter and measures degree of compression, and $b$ is the translation parameter that indicates the time location of the wavelet. If $|a|<1$ indicates the compressed version of the mother wavelet and is of higher frequency, then $|a|>1$ corresponds to lower frequencies [15].

Table 4. Definitions of statistical texture features

\begin{tabular}{|c|c|c|}
\hline $\begin{array}{l}\text { Order of } \\
\text { statistics }\end{array}$ & Texture features & Definitions \\
\hline \multirow[t]{7}{*}{$\begin{array}{r}\text { First-order } \\
\text { statistics }\end{array}$} & $\begin{array}{l}\text { Mean gray-level } \\
\text { intensity }\end{array}$ & $\begin{array}{l}\text { Average pixel value, i.e., intensity/ } \\
\text { brightness of a region }\end{array}$ \\
\hline & Standard deviation & Variation from mean gray-level value \\
\hline & & $\mathrm{SD}$ is small if image is homogenous \\
\hline & Uniformity & Uniformity of gray-level distribution \\
\hline & Entropy & Irregularity of gray-level distribution \\
\hline & Kurtosis & Flatness of histogram \\
\hline & Skewness & Asymmetry of histogram \\
\hline \multirow{6}{*}{$\begin{array}{l}\text { Second- } \\
\text { order } \\
\text { statistics }\end{array}$} & Local entropy & Measures randomness in image \\
\hline & & $\begin{array}{l}\text { Higher entropy indicates greater } \\
\text { randomness }\end{array}$ \\
\hline & Local homogeneity & $\begin{array}{l}\text { Measures closeness of distribution } \\
\text { of gray-level values in the matrix } \\
\text { (GLCM) to the GLCM diagonal }\end{array}$ \\
\hline & $\begin{array}{l}\text { Angular second } \\
\text { moment (ASM)/ } \\
\text { energy }\end{array}$ & $\begin{array}{l}\text { Measures homogeneity in an } \\
\text { image. Higher value indicates } \\
\text { greater uniformity of gray-level } \\
\text { values in a matrix }\end{array}$ \\
\hline & Dissimilarity & $\begin{array}{l}\text { Measurement of how different each } \\
\text { element in the matrix is }\end{array}$ \\
\hline & Correlation & $\begin{array}{l}\text { Measures gray-tone linear } \\
\text { dependencies }\end{array}$ \\
\hline \multirow{6}{*}{$\begin{array}{l}\text { Higher- } \\
\text { order } \\
\text { statistics }\end{array}$} & Coarseness & Measures the edge density \\
\hline & & Finer texture has higher edge density \\
\hline & Busyness & $\begin{array}{l}\text { Measures spatial rate of gray-level } \\
\text { change }\end{array}$ \\
\hline & Contrast & $\begin{array}{l}\text { Difference moment of the matrix, } \\
\text { measures local variations and } \\
\text { spread of matrix values }\end{array}$ \\
\hline & & $\begin{array}{l}\text { High contrast indicates greater } \\
\text { local variation, i.e., more } \\
\text { heterogeneous }\end{array}$ \\
\hline & Complexity & $\begin{array}{l}\text { Measures the amount of information } \\
\text { in an image (gray-level intensities, } \\
\text { number of sharp edges) }\end{array}$ \\
\hline
\end{tabular}

\section{References}

1 Ganeshan B, Miles KA, Young RC, Chatwin CR (2007) Hepatic entropy and uniformity: additional parameters that can potentially increase the effectiveness of contrast enhancement during abdominal CT. Clin Radiol, 62(8):761-768.

2 Ganeshan B, Abaleke S, Young RC, Chatwin CR, Miles KA (2010) Texture analysis of non-small cell lung cancer on unenhanced computed tomography: initial evidence for a relationship with tumour glucose metabolism and stage. Cancer Imaging, 10:137-143.

3 Miles KA, Ganeshan B, Griffiths MR, Young RC, Chatwin CR (2009) Colorectal cancer: texture analysis of portal phase hepatic CT images as a potential marker of survival. Radiology, 250(2):444-452.

4 Kato H, Kanematsu M, Zhang X, et al. (2007) Computer-aided diagnosis of hepatic fibrosis: preliminary evaluation of MRI texture analysis using the finite difference method and an artificial neural network. AJR Am J Roentgenol, 189(1):117-122.

5 Chen DR, Chang RF, Huang YL (1999) Computeraided diagnosis applied to US of solid breast nodules by using neural networks. Radiology, 213(2):407-412.

6 Smith TG, Jr., Lange GD, Marks WB (1996) Fractal methods and results in cellular morphologydimensions, lacunarity and multifractals. J Neurosci Methods, 69(2):123-136.

7 Sanghera B, Banerjee D, Khan A, et al. (2012) Reproducibility of 2D and 3D Fractal Analysis Techniques for the Assessment of Spatial Heterogeneity of Regional Blood Flow in Rectal Cancer. Radiology, 263 (3):865-873.

8 Plotnick RE, Gardner RH, Hargrove WW, Prestegaard K, Perlmutter M (1996) Lacunarity analysis: A general technique for the analysis of spatial patterns. Phys Rev E Stat Phys Plasmas Fluids Relat Interdiscip Topics, 53 (5):5461-5468.

9 Castellano G, Bonilha L, Li LM, Cendes F (2004) Texture analysis of medical images. Clin Radiol, 59 (12):1061-1069.

10 Zhang Y (2012) MRI texture analysis in multiple sclerosis. Int J Biomed Imaging, 2012:762804.

11 Brown RA, Frayne R (2008) A comparison of texture quantification techniques based on the Fourier and $\mathrm{S}$ transforms. Med Phys, 35(11):4998-5008.

12 Tuceryan M, Jain AK (1998) Texture analysis. In: Chen CH, Pau LF, Wang PSP (eds) The Handbook of Pattern Recognition and Computer Vision. World Scientific Publishing Co, Singapore, pp 207-248.

13 Arivazhagan S, Ganesan L (2003) Texture segmentation using wavelet transform. Pattern Recog Lett, 24:3197-3203. 
14 Mallat SG (1989) A theory of multiresolution signal decomposition: the wavelet representation. IEEE Transactions on Pattern Analysis and Machine Intelligence, 11(7):674-693.

15 Sifuzzaman M, Islam M, Ali M (2009) Application of wavelet transform and its advantages compared to Fourier transform. Journal of Physical Sciences, 13:121-137.

\section{References}

1. Hockel M, Knoop C, Schlenger K et al (1993) Intratumoral pO2 predicts survival in advanced cancer of the uterine cervix. Radiother Oncol 26(1):45-50

2. Yang Z, Tang LH, Klimstra DS (2011) Effect of tumor heterogeneity on the assessment of Ki67 labeling index in welldifferentiated neuroendocrine tumors metastatic to the liver: implications for prognostic stratification. Am J Surg Pathol 35(6):853860

3. Hockel M, Schlenger K, Aral B, Mitze M, Schaffer U, Vaupel P (1996) Association between tumor hypoxia and malignant progression in advanced cancer of the uterine cervix. Cancer Res 56 (19):4509-4515

4. Castellano G, Bonilha L, Li LM, Cendes F (2004) Texture analysis of medical images. Clin Radiol 59(12):1061-1069

5. Al-Kadi OS, Watson D (2008) Texture analysis of aggressive and nonaggressive lung tumor CE CT images. IEEE Trans Biomed Eng 55(7):1822-1830

6. Ganeshan B, Miles KA, Young RC, Chatwin CR (2007) Hepatic entropy and uniformity: additional parameters that can potentially increase the effectiveness of contrast enhancement during abdominal CT. Clin Radiol 62(8):761-768

7. Brown RA, Frayne R (2008) A comparison of texture quantification techniques based on the Fourier and S transforms. Med Phys 35(11):4998-5008

8. Goh V, Sanghera B, Wellsted DM, Sundin J, Halligan S (2009) Assessment of the spatial pattern of colorectal tumour perfusion estimated at perfusion CT using two-dimensional fractal analysis. Eur Radiol 19(6):1358-1365

9. Sanghera B, Banerjee D, Khan A et al (2012) Reproducibility of 2D and 3D Fractal Analysis Techniques for the Assessment of Spatial Heterogeneity of Regional Blood Flow in Rectal Cancer. Radiology 263(3):865-873

10. Craciunescu OI, Das SK, Clegg ST (1999) Dynamic contrastenhanced MRI and fractal characteristics of percolation clusters in two-dimensional tumor blood perfusion. J Biomech Eng 121 (5):480-486

11. Dettori L, Semler L (2007) A comparison of wavelet, ridgelet, and curvelet-based texture classification algorithms in computed tomography. Comput Biol Med 37(4):486-498

12. Al-Kadi OS (2010) Assessment of texture measures susceptibility to noise in conventional and contrast enhanced computed tomography lung tumour images. Comput Med Imaging Graph 34 (6):494-503

13. Tuceryan M, Jain AK (1998) Texture analysis. In: Chen CH, Pau LF, Wang PSP (eds) The Handbook of Pattern Recognition and Computer Vision. World Scientific Publishing Co, Singapore, pp 207-248

14. Amadasun M, King R (1989) Textural features corresponding to textural properties. IEEE Transactions on Systems, Man and Cybernetics 19:1264-1274
15. Srinivasan GN, Shobha G (2008) Statistical texture analysis. Proceedings of World Academy of Science, Engineering and Technology 36:1264-1269

16. Harlow CA, Eisenbeis SA (1973) The analysis of radiographic images. IEEE Trans Comput C22:678-689

17. Chen DR, Chang RF, Huang YL (1999) Computer-aided diagnosis applied to US of solid breast nodules by using neural networks. Radiology 213(2):407-412

18. Tourassi GD (1999) Journey toward computer-aided diagnosis: role of image texture analysis. Radiology 213(2):317-320

19. Eliat PA, Olivie D, Saikali S, Carsin B, Saint-Jalmes H, de Certaines JD (2012) Can dynamic contrast-enhanced magnetic resonance imaging combined with texture analysis differentiate malignant glioneuronal tumors from other glioblastoma? Neurol Res Int 2012:195176

20. Mayerhoefer ME, Schima W, Trattnig S, Pinker K, BergerKulemann V, Ba-Ssalamah A (2010) Texture-based classification of focal liver lesions on MRI at 3.0 Tesla: a feasibility study in cysts and hemangiomas. J Magn Reson Imaging 32 (2):352-359

21. Lopes R, Ayache A, Makni N et al (2011) Prostate cancer characterization on MR images using fractal features. Med Phys 38 (1):83-95

22. Holli K, Laaperi AL, Harrison L et al (2010) Characterization of breast cancer types by texture analysis of magnetic resonance images. Acad Radiol 17(2):135-141

23. Zacharaki EI, Wang S, Chawla S et al (2009) Classification of brain tumor type and grade using MRI texture and shape in a machine learning scheme. Magn Reson Med 62(6):1609-1618

24. Alic L, van Vliet M, van Dijke CF, Eggermont AM, Veenland JF, Niessen WJ (2011) Heterogeneity in DCE-MRI parametric maps: a biomarker for treatment response? Phys Med Biol 56(6): $1601-1616$

25. Assefa D, Keller H, Menard C, Laperriere N, Ferrari RJ, Yeung I (2010) Robust texture features for response monitoring of glioblastoma multiforme on T1-weighted and T2-FLAIR MR images: a preliminary investigation in terms of identification and segmentation. Med Phys 37(4):1722-1736

26. Karahaliou A, Vassiou K, Arikidis NS, Skiadopoulos S, Kanavou T, Costaridou L (2010) Assessing heterogeneity of lesion enhancement kinetics in dynamic contrast-enhanced MRI for breast cancer diagnosis. Br J Radiol 83(988):296-309

27. Harrison LC, Luukkaala T, Pertovaara H et al (2009) Non-Hodgkin lymphoma response evaluation with MRI texture classification. J Exp Clin Cancer Res 28:87

28. O'Connor JP, Rose CJ, Jackson A et al (2011) DCE-MRI biomarkers of tumour heterogeneity predict CRC liver metastasis shrinkage following bevacizumab and FOLFOX-6. Br J Cancer 105(1):139-145

29. Rose CJ, Mills SJ, O'Connor JP et al (2009) Quantifying spatial heterogeneity in dynamic contrast-enhanced MRI parameter maps. Magn Reson Med 62(2):488-499

30. El Naqa I, Grigsby P, Apte A et al (2009) Exploring feature-based approaches in PET images for predicting cancer treatment outcomes. Pattern Recognit 42(6):1162-1171

31. Tixier F, Le Rest CC, Hatt M et al (2011) Intratumor heterogeneity characterized by textural features on baseline 18 F-FDG PET images predicts response to concomitant radiochemotherapy in esophageal cancer. J Nucl Med 52(3):369-378

32. Ganeshan B, Burnand K, Young R, Chatwin C, Miles K (2011) Dynamic contrast-enhanced texture analysis of the liver: initial assessment in colorectal cancer. Invest Radiol 46(3): $160-168$

33. Ganeshan B, Skogen K, Pressney I, Coutroubis D, Miles K (2012) Tumour heterogeneity in oesophageal cancer assessed by $\mathrm{CT}$ 
texture analysis: preliminary evidence of an association with tumour metabolism, stage, and survival. Clin Radiol 67(2):157-164

34. Ganeshan B, Abaleke S, Young RC, Chatwin CR, Miles KA (2010) Texture analysis of non-small cell lung cancer on unenhanced computed tomography: initial evidence for a relationship with tumour glucose metabolism and stage. Cancer Imaging 10:137-143

35. Ganeshan B, Goh V, Mandeville H, Ng QS, Hoskin P, Miles KA (In press) CT of non-small cell lung cancer: Histopathological correlates for texture parameters. Radiology.

36. Kido S, Kuriyama K, Higashiyama M, Kasugai T, Kuroda C (2003) Fractal analysis of internal and peripheral textures of small peripheral bronchogenic carcinomas in thin-section computed tomography: comparison of bronchioloalveolar cell carcinomas with nonbronchioloalveolar cell carcinomas. J Comput Assist Tomogr 27(1):56-61

37. Kido S, Kuriyama K, Higashiyama M, Kasugai T, Kuroda C (2002) Fractal analysis of small peripheral pulmonary nodules in thin-section CT: evaluation of the lung-nodule interfaces. J Comput Assist Tomogr 26(4):573-578

38. Huang YL, Chen JH, Shen WC (2006) Diagnosis of hepatic tumors with texture analysis in nonenhanced computed tomography images. Acad Radiol 13(6):713-720

39. Cui C, Cai H, Liu L, Li L, Tian H (2011) Quantitative analysis and prediction of regional lymph node status in rectal cancer based on computed tomography imaging. Eur Radiol 21(11):2318-2325

40. Skogen K, Ganeshan B, Good T, Critchley G, Miles KA (2011) Imaging heterogeneity in gliomas using texture analysis. Cancer Imaging, 11 Spec No A:S113.

41. Goh V, Ganeshan B, Nathan P, Juttla JK, Vinayan A, Miles KA (2011) Assessment of response to tyrosine kinase inhibitors in metastatic renal cell cancer: CT texture as a predictive biomarker. Radiology 261(1):165-171

42. Ganeshan B, Miles KA, Young RC, Chatwin CR (2007) In search of biologic correlates for liver texture on portal-phase CT. Acad Radiol 14(9):1058-1068

43. Miles KA, Ganeshan B, Griffiths MR, Young RC, Chatwin CR (2009) Colorectal cancer: texture analysis of portal phase hepatic CT images as a potential marker of survival. Radiology 250(2):444-452

44. Ganeshan B, Panayiotou E, Burnand K, Dizdarevic S, Miles K (2012) Tumour heterogeneity in non-small cell lung carcinoma assessed by CT texture analysis: a potential marker of survival. Eur Radiol 22(4):796-802
45. James D, Clymer BD, Schmalbrock P (2001) Texture detection of simulated microcalcification susceptibility effects in magnetic resonance imaging of breasts. J Magn Reson Imaging 13(6):876-881

46. Gibbs P, Turnbull LW (2003) Textural analysis of contrastenhanced MR images of the breast. Magn Reson Med 50(1):92-98

47. Chen W, Giger ML, Li H, Bick U, Newstead GM (2007) Volumetric texture analysis of breast lesions on contrastenhanced magnetic resonance images. Magn Reson Med 58 (3):562-571

48. Woods BJ, Clymer BD, Kurc T et al (2007) Malignant-lesion segmentation using $4 \mathrm{D}$ co-occurrence texture analysis applied to dynamic contrast-enhanced magnetic resonance breast image data. J Magn Reson Imaging 25(3):495-501

49. Lv D, Guo X, Wang X, Zhang J, Fang J (2009) Computerized characterization of prostate cancer by fractal analysis in MR images. J Magn Reson Imaging 30(1):161-168

50. Eary JF, O'Sullivan F, O'Sullivan J, Conrad EU (2008) Spatial heterogeneity in sarcoma 18 F-FDG uptake as a predictor of patient outcome. J Nucl Med 49(12):1973-1979

51. Yu H, Caldwell C, Mah K, Mozeg D (2009) Coregistered FDG PET/CT-based textural characterization of head and neck cancer for radiation treatment planning. IEEE Trans Med Imaging 28 (3):374-383

52. Yu H, Caldwell C, Mah K et al (2009) Automated radiation targeting in head-and-neck cancer using region-based texture analysis of PET and CT images. Int J Radiat Oncol Biol Phys 75 (2):618-625

53. Mayerhoefer ME, Szomolanyi P, Jirak D, Materka A, Trattnig S (2009) Effects of MRI acquisition parameter variations and protocol heterogeneity on the results of texture analysis and pattern discrimination: an application-oriented study. Med Phys 36 (4): $1236-1243$

54. Galavis PE, Hollensen C, Jallow N, Paliwal B, Jeraj R (2010) Variability of textural features in FDG PET images due to different acquisition modes and reconstruction parameters. Acta Oncol 49 (7):1012-1016

55. Waugh SA, Lerski RA, Bidaut L, Thompson AM (2011) The influence of field strength and different clinical breast MRI protocols on the outcome of texture analysis using foam phantoms. Med Phys 38(9):5058-5066

56. Collewet G, Strzelecki M, Mariette F (2004) Influence of MRI acquisition protocols and image intensity normalization methods on texture classification. Magn Reson Imaging 22(1):81-91 